\title{
DYNAMIQUE D'UNE POPULATION EXPÉRIMENTALE DE BRACHIONUS DIMIDIATUS (BRYCE) (ROTIFẼRE) EN FONCTION DE LA NOURRITURE ET DE LA TEMPÉRATURE
}

\author{
par R. Pourriot ${ }^{1}$ et Cl. Rougier ${ }^{1}$.
}

\begin{abstract}
La concentration du milieu en carbonates alcalins, entre 1 et $18 \mathrm{~g}^{-1}$, n'affecte pas le taux intrinsèque d'accroissement naturel de $B$. dimidiatus qui dépend essentiellement de la température et de la qualité de la nourriture offerte. Les taux de croissance les plus élevés sont obtenus avec les Cyanophycées Chroococcales (Synechococcus cedrorum) ou des Chlorophycées Volvocales (Dunaliella salina) unicellulaires de petites tailles. La température agit peu sur le taux net de reproduction et le fort taux intrinsèque d'accroissement naturel à $30^{\circ}$ est dû au raccourcissement des temps de développement embryonnaire et post-embryonnaire ainsi qu'à la rapidité du rythme de ponte.
\end{abstract}

\section{The dynamic}

\section{of a laboratory population of Brachionus dimidiatus (Bryce) (Rotatoria) in relation to food and temperature.}

Concentrations of $\mathrm{NaHCO}_{3}$ in the water, between 1 and $18 \mathrm{~g}^{-1}$ do not affect the intrinsic rate of natural increase in populations of $\boldsymbol{B}$. dimidiatus. This rate depends chiefly on temperature and food quality. The highest rates of growth were obtained with unicellular blue-green algae (Synechoccus cedro'rum) or green algae (Dunaliella salina). The influence of temperature on the life span, fecundity, egg development and minimum generation time was established at 20,25 and $30^{\circ} \mathrm{C}$. Temperature has little effect on the net reproductive rate. The high intrinsic rate of natural increase at $30^{\circ} \mathrm{C}$ is due to reduced embryonic and post-embryonic development times and also to the speed of the rhythm of egg laying.

Brachionus dimidiatus Bryce est un petit Brachionide (Rotifère) provenant des mares natronées du Kanem au nord du Tchad. Ces mares sont souvent temporaires, installées dans les dépressions du système dunaire fossile caractéristique du Kanem, et alimentées par les eaux de pluies et de la nappe phréatique (Iltis 1969 ; Iltis et Riou-Du'wat 1971). La particularité essentielle de ce biotope est

1. Laboratoire de Génétique Evolutive et de Biométrie, C.N.R.S., 91190 Gifsur-Yvette.

Mots-clefs :

Dynamique de population. Taux de croissance-nourriture. Taux de croissance-température. Rotifères. Brachionus. 
sa teneur élevée en carbonates et bicarbonates alcalins (cf. Pourriot, Iltis, Levêque-Duwat 1967). Le $\mathrm{pH}$ des eaux varie entre 9 et 10,4 u. $\mathrm{pH}$ et la température reste toujours assez élevée, le minimum absolu étant de 14 à $15^{\circ} \mathrm{C}$ et le maximum $35^{\circ} \mathrm{C}$.

Les populations de $B$. dimidiatus (qui se multiplient parthénogénétiquement) atteignent dans ces mares des densités très élevées (ainsi, dans la mare de Latir, la densité de $B$. dimidiatus peut atteindre plus de 600 individus par $\mathrm{ml}$ ). Après les observations faites par Iltis et Riou-Duwat (1971) sur des populations naturelles, il s'avérait intéressant d'étudier la dynamique d'une population expérimentale et les paramètres biologiques liés à cette population : durée de développement embryonnaire et postembryonnaire et temps de génération.

Le taux de croissance de la population dépend, bien entendu, des conditions d'élevage. L'étude de ce taux et de l'influence de la température sera précédée d'observations préliminaires dans le but de déterminer les conditions optimales d'élevage : concentration saline du milieu et qualité de la nourriture.

\section{I. - OBSERVATIONS PRÉLIMINAIRES}

\section{a) MATÉriel ET MÉthodes.}

La souche de $B$. dimidiatus élevée au laboratoire depuis décembre 1971 a été isolée à partir d'une récolte de plancton faite par A. Iltis dans un ouadi de Djikare $\left(13^{\circ} 53 \mathrm{~N}-14^{\circ} 13^{\prime} 30^{\prime \prime} \mathrm{E}\right)$. La composition du milieu de culture appelé $\mathrm{Na}_{6}$ (tableau I), élaboré au laboratoire pour les algues (Cyanophycées) provenant de ces biotopes, s'inspire, bien entendu, des caractères physicochimiques de ces derniers. La composition du milicu usuel d'élevage pour les brachions dérive du précédent $\left(\mathrm{Na}_{6}\right.$ dilué au $\left.1 / 4\right)$.

L'algue utilisée comme nourriture est la Cyanophyte Synechococcus cedrorum (Chroococcale).

\begin{tabular}{ll}
\hline $\mathrm{Na} \mathrm{H} \mathrm{CO}_{3}$ & $=18 \mathrm{~g}$ \\
$\mathrm{~K}_{2} \mathrm{H} \mathrm{PO}_{4}$ & $=300 \mathrm{mg}$ \\
$\mathrm{K} \mathrm{NO}_{3}$ & $=500 \mathrm{mg}$ \\
$\mathrm{K}_{2} \mathrm{SO}_{4}$ & $=1 \mathrm{~g}$ \\
$\mathrm{Na} \mathrm{Cl}$ & $=1 \mathrm{~g}$ \\
$\mathrm{Ca} \mathrm{Cl}_{2}, 2 \mathrm{H}_{2} \mathrm{O}$ & $=40 \mathrm{mg}$ \\
$\mathrm{Mg} \mathrm{Cl}_{2}, 6 \mathrm{H}_{2} \mathrm{O}$ & $=20 \mathrm{mg}$ \\
$\mathrm{Na}_{2} \mathrm{SiO}_{3,} 5 \mathrm{H}_{2} \mathrm{O}$ & $=250 \mathrm{mg}$ \\
\hline
\end{tabular}

Tableau I. - Composition du milieu de culture « $\mathrm{Na}_{\mathrm{f}} »$ pour algues d'eaux natronées.

Composition of the culture medium « $\mathrm{Na}_{\mathrm{q}}$ 》 for natron lake algae. 
Pour chaque expérience, 20 femelles ovigères amictiques de $B$. dimidiatus sont placées dans une boîte de Pétri contenant le milieu approprié et de la nourriture toujours en léger excès. La boîte de Pétri est mise dans une chambre humide, elle-même plongée dans un bain thermostaté (cf. Pourriot et Hillbricht Ilkowska 1969). Leur descendance est dénombrée toutes les 24 heures. 'La reproduction est parthénogénétique, il n'y a pas d'intervention de la reproduction sexuée. Le dénombrement est arrêté dès l'apparition de quelques mâles, qui se situe en fin d'expérience, lorsque le nombre d'individus devient très élevé (25 à 30 ind./ml). L'accroissement d'une telle population est de type exponentiel ; en reportant sur papier semi-logarithmique, le nombre d'individus comptés chaque jour, on obtient une droite dont la pente calculée représente le taux d'accroissement désigné par le symbole $r$ (= taux intrinsèque d'accroissement: voir plus loin). Les différentes valeurs prises par $r$ permettent de comparer l'influence des facteurs étudiés sur l'accroissement de la population.

\section{b) INFLUENCE de LA CONCENTRATION DU MiliEu.}

Plusieurs concentrations du milieu « $\mathrm{Na}_{6}$ » ont été étudiées afin de voir celle (s) qui convenait (ent) le mieux aux $B$. dimidiatus. Les expériences sont toutes faites à $20 \pm 0,5^{\circ} \mathrm{C}$, le $\mathrm{pH}$ des différents milieux étant $9 \pm 0,1$ u. $\mathrm{pH}$ et les Rotifères nourris avec Synechococcus cedrorum.

\begin{tabular}{cccccc}
\hline & $\mathrm{Na}_{6}$ & $\mathrm{Na}_{6} 1 / 2$ & $\mathrm{Na}_{6} 1 / 4$ & $\mathrm{Na}_{6} 1 / 8$ & $\mathrm{Na}_{6} 1 / 16$ \\
\hline $\mathrm{r}$ & $\mathbf{0 , 4 1 7}$ & $\mathbf{0 , 4 2 4}$ & 0,419 & 0,417 & 0,429 \\
\hline
\end{tabular}

TABLEAU II. - Taux intrinsèques d'accroissement (r) de $B$. dimidiatus à différentes concentrations du milieu « $\mathrm{Na}_{\text { }} 》$ à $20^{\circ} \mathrm{C}$.

Intrinsic rates of natural increase ( $\mathrm{r}$ ) of $B$. dimidiatus at different concentrations of the « $\mathrm{Na}_{6}$ » medium.

Les différentes concentrations utilisées sont : $\mathrm{Na}_{6}, \mathrm{Na}_{6}$ dilué au $1 / 2$, au $1 / 4$, au $1 / 8$ el au $1 / 16$. La dilution au $1 / 4$ correspond à celle du milieu usuel d'élevage de ce Rotifère au laboratoire.

Alors qu'il a fallu adapter progressivement les animaux au milieu « $\mathrm{Na}_{6}$ » non dilué, les brachions se sont très bien adaptés aux différentes concentrations en carbonates et, dans tous les cas, le taux de croissance de la population a été sensiblement le même (tableau II et fig. 1).

Ce fait est en rapport avec les conditions de vie de ces animaux qui, dans leur milieu naturel (mares natronées), sont soumis à de fortes variations de la concentration du milieu dont « la teneur en sels décroît durant la phase de mise en eau en juillet, août et 

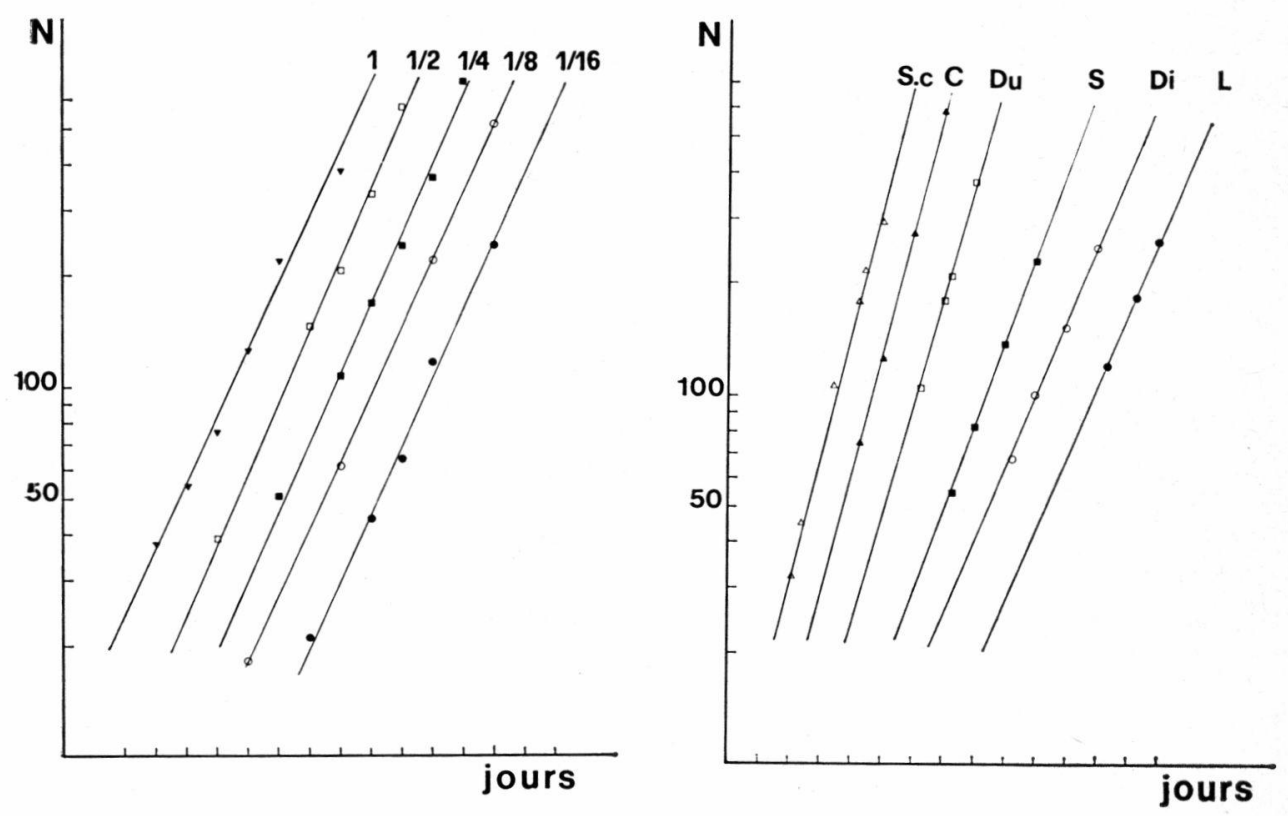

FIg. 1. - Courbes de croissance de $B$. dimidiatus à différentes dilutions du milieu $\mathrm{Na} 6$, à $20^{\circ} \mathrm{C}$.

Growth curves of $B$. dimidiatus at different dilutions of the $\mathrm{Na} 6$ medium.

FIG. 2. - Courbes de croissance de $B$. dimidiatus avec différentes espèces nutritives, à $25^{\circ} \mathrm{C}$ : Synechococcus cedrorum (S.c.), Synechocystis sp (S), Chlamydomonas sp (C), Dunaliella salina (Du), Diogenes sp (Di) et levure (L.).

Growth curves of $B$. dimidiatus with different food species, at $25^{\circ} \mathrm{C}$.

septembre, puis augmente au début de la saison sèche » (Iltis et Riou-Duwat 1971). B. dimidiatus est signalé par ces derniers auteurs dans des eaux à salinité élevée (résidu sec à $105^{\circ}=70 \mathrm{~g} \mathbf{1}^{-1}$ ) mais aussi dans des mares à faible concentration saline (résidu $\sec =1,65 \mathrm{~g}^{-1}$ ). Les expériences au laboratoire confirment bien les observations faites in situ : B. dimidiatus est, parmi les autres Rotifères de milieu hypersalin, l'espèce ayant les exigences les moins strictes du point de vue tolérance à la salinité.

Rut!ner-Kolisko (1972) indique que B. plicatilis, en milieu natroné, montre au contraire une préférence pour les faibles concentrations.

c) INFLUENCE DE LA QUALITÉ DE LA NOURRITURE.

Maintenu au laboratoire en présence d'Oscillatoria platensis lors des premiers essais en $1963, B$. dimidiatus ne se nourrissait pas de ces Cyanophycées filamenteuses et spiralées (donc non ingérables par le brachion) mais plus vraisemblablement des produits 


\begin{tabular}{|c|c|c|c|c|c|c|c|}
\hline EsPÈCES & 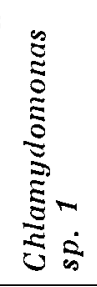 & 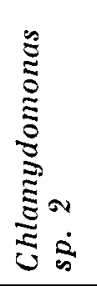 & 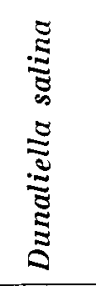 & 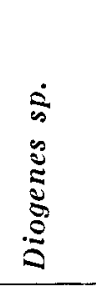 & 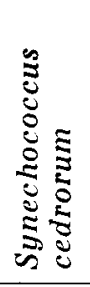 & 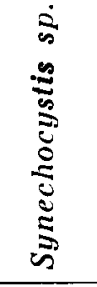 & 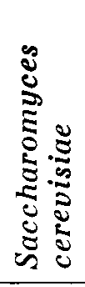 \\
\hline 20 & 0,507 & - & 0,525 & 0,380 & 0,460 & 0,470 & 0,300 \\
\hline 25 & - & 0,734 & 0,700 & 0,430 & 0,740 & 0,520 & 0,420 \\
\hline
\end{tabular}

Tableau III. - Différentes valeurs du taux intrinsèque d'accroissement en fonction de la qualité de la nourriture, aux températures de 20 et $25^{\circ} \mathrm{C}$. Various values of the intrinsic rate of natural increase in relation to the food quality and the temperature.

de décomposition et des Bactéries présentes dans le milieu. Le problème de leur alimentation n'avait alors pas pu être résolu complètement.

La deuxième séric d'essais d'élevage effectuéc en 1971 a été plus fructueuse puisque $B$. dimidiatus s'est multiplié en présence de plusieurs espèces d'algues et même de levure, quoique à un moindre degré. Les espèces suivantes ont été proposécs comme nourriture aux $B$. dimidiatus : Dunaliella salina, Chlamydomonas $s p$., Diogenes sp. (Chlorophytes), Synechocystis sp., Synechococcus cedrorum (Cyanophytes) ainsi que la levure Saccharomyces cerevisiae.

Les différentes valeurs du taux intrinsèque d'accroissement obtenu avec chacune de ces espèces ont été calculées (tableau III et fig. 2) d'après les effectifs d'une population en croissance exponentielle.

Les Cyanophycées Chroococcales et les Chlorophycées Volvocales unicellulaires fournissent les taux d'accroissement les plus élevés. Le faible taux de croissance obtenu à $25^{\circ}$ avec Synechocystis sp. (cellules isolées contenant de nombreuses vacuoles gazcuscs) pourrait provenir d'un antagonisme dû à des substances extracellulaires excrétées par la Cyanophycée (cf. Pourriot 1966).

Tous ces résultats indiquent que $B$. dimidiatus est un microphage typique, ne pouvant se nourrir que de fines particules $(\leqslant 25 \mu)$ et que des différences dans le régime alimentaire peuvent apparaître d'une souche à une autre. Cette constatation n'est pas surprenante puisque des observations identiques ont été faites sur d'autres espèces planctoniques (cf. Pourriot 1965) et confirme l'existence de variations génétiques dans les populations (King 1972).

Dans les mares natronées où prédominent parfois les Flagellés verts et le plus souvent des Cynanophycées, B. dimidiatus pourra 
se nourrir de Volvocales unicellulaires (Chlamydomonas) et des petites Chroococcales (Synechococcus, Synechocystis, etc.) ainsi que des fins détritus provenant de la décomposition des Cyanophytes filamenteuses et des Bactéries associées.

\section{II. - ETUDE DE QUELQUES PARAMETRES BIOLOGIQUES}

Les différents paramètres éludiés sur les femelles amictiques de B. dimidiatus sont :

- De $=$ durée de développement de l'embryon,

- $\mathrm{Dp}=$ durée de développement post-embryonnaire,

- $\mathrm{Tg}=$ temps minimum séparant deux générations.

La durée du développement embryonnaire est évalué par la méthode simplifiée d'Edmondson (1965), exposée par Pourriot et Deluzarches (1971). En outre, à $20^{\circ}$, des mesures directes de ce temps de développement sont faites. La comparaison de ces deux

\begin{tabular}{|c|c|c|c|c|c|}
\hline Températur & e en ${ }^{\circ} \mathrm{C}$ & 20 & & 25 & 30 \\
\hline \multirow[t]{4}{*}{$\begin{array}{c}\text { Synechococcus } \\
\text { cedrorum }\end{array}$} & De & $\begin{array}{l}\text { 1) } 25^{*} \\
\text { 2) } 24,2 \pm 4,0\end{array}$ & (8) & $14,3^{*}$ & $10,5^{*}$ \\
\hline & $\mathrm{Dp}$ & $46,1 \pm 2,6$ & $(8)$ & $25,5 \pm 3,0$ & 12,4 (calculé) \\
\hline & $\mathrm{Tg}$ & $68,4 \pm 1,5$ & (8) & $40,0 \pm 1,3$ & $22,9 \pm 3,2$ \\
\hline & $\mathrm{Dp} / \mathrm{De}$ & 1,8 & & 1,8 & 1,2 \\
\hline \multirow[t]{4}{*}{$\begin{array}{l}\text { Dunaliella } \\
\text { salina }\end{array}$} & De & $\begin{array}{l}\text { 1) } 25^{*} \\
\text { 2) } 24 \pm 4\end{array}$ & & $14,3^{*}$ & $10,5^{*}$ \\
\hline & Dp & $45,7 \pm 2,9$ & (6) & $23,5 \pm 1,4$ & 12,3 (calculé) \\
\hline & $\mathrm{Tg}$ & $68,4 \pm 2,6$ & (4) & $39,7 \pm 0,6$ & $22,8 \pm 1,2$ \\
\hline & $D_{p} / D_{e}$ & 1,8 & & 1,7 & 1,2 \\
\hline
\end{tabular}

Tableau IV. - Valeurs de différents paramètres biologiques obtenues pour des femelles amictiques de $B$. dimidiatus, à 3 températures et avec 2 nourritures différentes. Les résultats sont exprimés en heures et en fractions décimales. Le nombre d'individus expérimentés est indiqué entre parenthèses. Les résultats suivis d'un astérisque ont été obtenus avec la méthode simplifiée.

Values in hours of $\mathrm{De}, \mathrm{Dp}$ et $\mathrm{Tg}$ for amictic females of $B$. dimidiatus, at 3 temperatures and with 2 food species : $M \pm$ e.t. (n).

méthodes montre que les résultats obtenus sont tout à fait comparables (tabl. IV). Les autres paramètres sont mesurés directement selon les méthodes décrites par Pourriot et HillbrichtIlkowska (1969). 

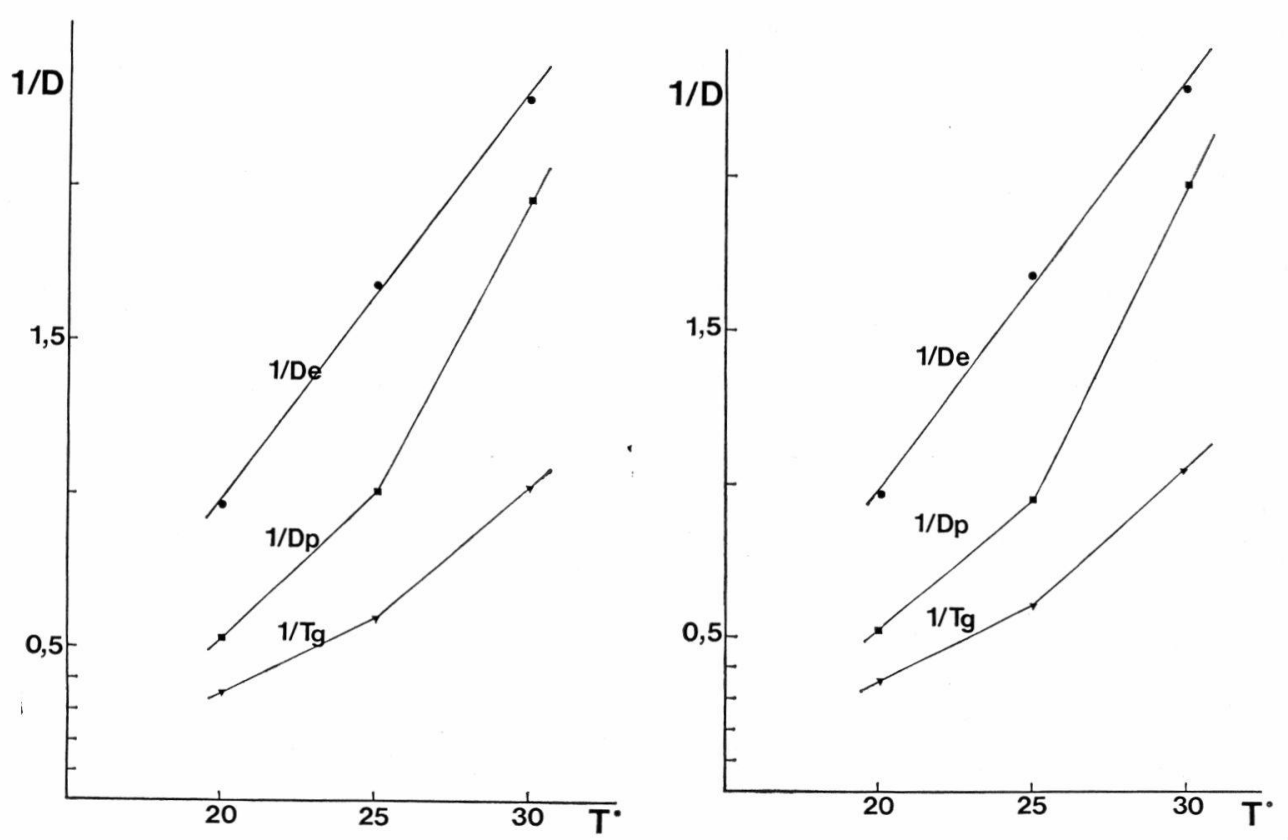

Fig. 3. - Variation de $1 / \mathrm{De}, 1 / \mathrm{Dp}$ et $1 / \mathrm{Tg}$ en fonction de la température chez $B$. dimidiatus nourris de Synechococcus cedrorum (a) ou de Dunaliella salina (b).

Variation of $1 / \mathrm{De}, 1 / \mathrm{Dp}$ et $1 / \mathrm{Tg}$ in relation to temperature in $B$. dimidia tus feeding on $S$. cedrorum (a) or D. salina (b).

Habituellement, il plus aisé de suivre la variation du taux de développement par unité de temps, c'est-à-dire l'inverse de la durée de développement en fonction de la température. Edmondson (1960-1965) et Amren (1964) ont montré que chez les Rotifères, cette relation était linéaire entre certains intervalles de température : $1 / \mathrm{D}=\mathrm{a} t+\mathrm{b}$. Par souci d'homogénéité avec d'autres travaux portant notamment sur les Crustacés (Gras et Saint-Jean 1969), l'unité de temps considérée ci-dessous n'est pas l'heure, mais le jour.

\begin{tabular}{cccc}
\hline $\begin{array}{c}\text { Ecart de } \\
\text { température }\end{array}$ & $\mathrm{Q}_{10}$ de $1 / \mathrm{De}$ & $\mathrm{Q}_{10}$ de $1 / \mathrm{Dp}$ & $\mathrm{Q}_{10}$ de $1 / \mathrm{Tg}$ \\
\hline $25-25^{\circ}$ & 2,86 & 3,27 & 2,94 \\
\hline $25-30^{\circ}$ & 1,84 & 4,35 & 3,09 \\
\hline
\end{tabular}

Tableau V. - Valeurs du $Q_{10}$ pour les taux de développement embryonnaire et post-embryonnaire et l'inverse du temps de génération.

$Q_{1 n}$ values for the rate of embryonic development, post-embryonic development and $1 / \mathrm{Tg}$ (generation time). 
En premier lieu, il faut souligner la similitude des résulta!s obtenus avec les deux espèces algales : la différence existant à $25^{\circ}$ entre les durées de développement post-embryonnaire n'est pas significative. La valeur nutritive pour $B$. dimidiatus de ces deux espèces d'algues appartenant à deux classes bien différentes : Chlorophycée et Cyanophycée, est donc semblable.

La représentation graphique des variations de $1 / \mathrm{De}, 1 / \mathrm{Dp}$ et $1 / \mathrm{Tg}$ en fonction de la température (fig. 3 a et b) indique que seul 1/De est directement proportionnel à la température, l'équation de la courbe obtenue étant $1 / \mathrm{De}=0,130 \quad t-1,61$ alors que pour 13 espèces de Rotifères, Pourriot el Deluzarches (1971) avaient ob!enu également pour le développement post-embryonnaire et donc pour le temps de génération, les équations du type $1 / \mathrm{D}=\mathrm{f}(\mathrm{t})$.

Cependant, la courbe représentative de $1 / \mathrm{Tg}$ en fonction de la température, tracée par Pourriot et Deluzarches (1971) d'après les résultats d'Halbach (1970 a) sur le Rotifère Brachionus calyciflorus, présente à $20^{\circ} \mathrm{C}$ une rupture de pente. A partir de cette température, la pente devient nettement plus forte. Chez $B$. dimidiatus, la pente des droites représentant $1 / \mathrm{Dp}$ et $1 / \mathrm{Tg}$ en fonction de la température est plus élevée entre 25 et $30^{\circ} \mathrm{C}$ qu'entre 20 et $25^{\circ} \mathrm{C}$. La température où a lieu la rupture de pente n'est pas la même pour les deux espèces de Brachionus, mais l'allure des courbes est comparable et clle traduit vraisemblablement le fait que les algues (Synechococcus et Dunaliella pour B. dimidiatus et Chlorelles pour $B$. calyciflorus) sont mieux utilisèes aux températures élevées.

Les $Q_{10}$ ont été calculés entre 20 et $25^{\circ} \mathrm{C}$ et, 25 et $30^{\circ} \mathrm{C}$, pour les différents taux $(1 / \mathrm{De}, 1 / \mathrm{Dp}$ et $1 / \mathrm{Tg})$. Les résultats sont les mêmes avec les 2 espèces algales servant de nourriture (tableau V).

Le $Q_{10}$ de $1 / D e$ est plus faible entre $25-30{ }^{\circ} \mathrm{C}$ qu'entre 20 et $25{ }^{\circ} \mathrm{C}$. Chez les poïkilothermes on observe en effet généralement une diminution du $Q_{10}$ avec l'augmentation de la lempérature (Rao et Bullock 1954). Cependant pour le développement postembryonnaire, c'est l'effet inverse qui se produit, mais, comme il a déjà été indiqué, seul le développement est directement et uniquement influencé par la température. Quant au temps de génération qui intègre les deux autres paramètres, la valeur du $Q_{10}$ pour les deux écarts de température est sensiblement la même.

Enfin, le rapport Dp/De, approximativement constant chez les Cladocères (Gras et Saint-Jean 1969) et peu variable chez les Rotifères (Pourriot et Deluzarches 1971) est dans ces expériences sur $B$. dimidiatus nettement inférieur, à $30^{\circ} \mathrm{C}$, à celui obtenu aux 2 autres températures (tableau IV). Ceci traduit le fait que les développements embryonnaires et post-embryonnaires n'ont pas été accélérés de la même manière par l'augmentation de la 
température : à $30^{\circ} \mathrm{C}$ le temps de ponte de l'œuf est proportionnellement plus rapide que la durée d'éclosion.

\section{III. - ETUDE DE LA CROISSANCE DE LA POPULATION}

La connaissance du taux net de reproduction Ro, (facteur par lequel se multiplie la population d'une génération à l'autre) et celle du nombre de descendants par femelle et par jour permettent de calculer le taux intrinsèque d'accroissement naturel $r$. Pour évaluer ces facteurs, la descendance de jeunes femelles isolées a été suivie quotidiennement jusqu'à leur mort ; les jeunes femelles écloses étaient dénombrées et enlevées à chaque observation.

\subsection{Taux de fécondité et taux net de reproduction.}

Sur les histogrammes de répartition des descendants en fonction de l'âge de la mère (fig. 4), le nombre de jeunes produits chaque jour est rangé par classe (la classe I correspond à la production de 1 et 2 individus, la classe II à celle de 3 et 4 individus, la classe III à celle de 5 et 6 individus et la classe IV à celle de 7 et 8 individus).

A l'augmentation de la température, correspond une augmentation du nombre de jeunes produits par femelle et par jour, dès les premiers jours de vie de la $q$ mère et une diminution de l'intervalle entre chaque ponte. A $30^{\circ} \mathrm{C}$, apparaît la classe IV ( 7 à 8 jeunes $/ q / \mathrm{j}$ dès le $2^{c}$ jour de vie de la mère), qui n'existait ni à $25^{\circ} \mathrm{C}$, ni bien sûr à $20^{\circ} \mathrm{C}$, où ne figurent que les deux premières classes.

Dans le tableau VI a et $b$ sont fournies les différentes valeurs prises aux trois températures par les paramètres étudiés, à savoir: les taux de survie $\left(l_{x}\right)$ et de fécondité $\left(m_{x}=n\right.$ descendants/ $/$ j) d'une femelle d'âge $x$ ainsi que leur produit $\left(l_{x} m_{x}\right)$. Ce dernier correspond au nombre de descendants produits par jour et par femelle initiale.

L'évolution des taux de survie est similaire aux trois températures et avec les deux nourritures (fig. 5 a et b).

L'évolution de la fécondité en fonction de l'âge de la mère (fig. 6 a et b) montre des variations identiques quelle que soit la nourriture utilisée. L'allure des courbes obtenues à 20 et $25^{\circ} \mathrm{C}$ n'est pas très différente, la seconde étant légèrement au-dessus de la première. Mais la courbe de fécondité à $30^{\circ} \mathrm{C}$ diffère nettement dans les deux cas et présente un pic très marqué suivi d'une chute rapide. 

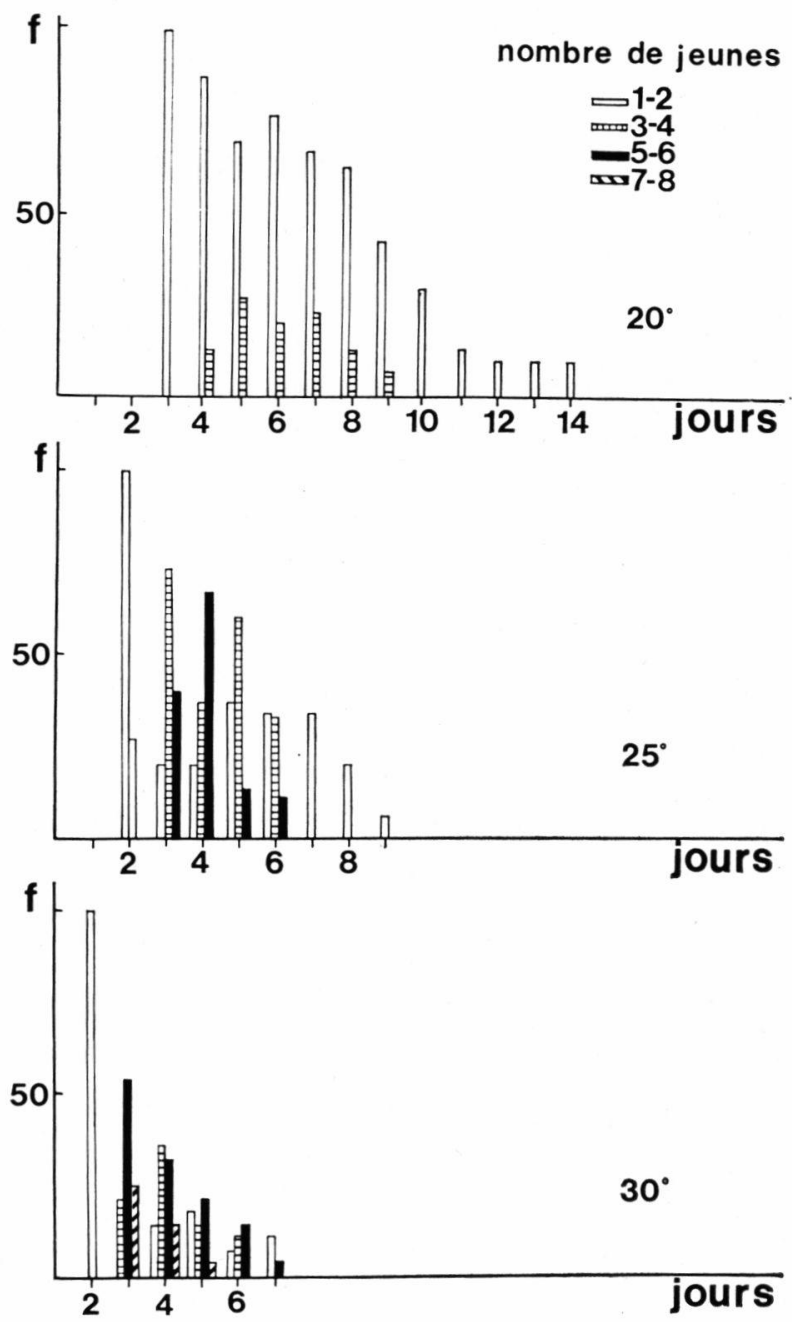

Fig. 4. - Histogrammes de fréquence montrant la répartition des descendants en fonction de l'âge de la mère et de la température chez $B$. dimidiatus.

Frequency histograms showing the offsprings distribution in relation to the maternal age and to the temperature in $B$. dimidiatus.

Le taux net de reproduction correspond au nombre d'œufs pondus pendant la durée de vie de femelle amictique. Or l'augmentation de la température diminue non seulement la durée des développements embryonnaires et post-embryonnaires, mais aussi la durée de vie moyenne, et par conséquent la période de maturité sexuelle est raccourcie. A $20^{\circ} \mathrm{C}$, des individus nourris avec Dunaliella ou Synechococcus vivent en moyenne une huitaine 


\begin{tabular}{|c|c|c|c|c|c|c|c|c|c|}
\hline \multirow{2}{*}{$\begin{array}{c}\text { Age en } \\
\text { jours }(x)\end{array}$} & \multicolumn{3}{|c|}{$20^{\circ}$} & \multicolumn{3}{|c|}{$25^{\circ}$} & \multicolumn{3}{|c|}{$30^{\circ}$} \\
\hline & $1_{x}$ & $m_{x}$ & $1_{\times} m_{x}$ & $1_{x}$ & $m_{x}$ & $1_{\times} m_{x}$ & $1_{x}$ & $\mathrm{~m}_{\mathrm{x}}$ & $1_{\mathrm{x}} \mathrm{m}_{\mathrm{x}}$ \\
\hline 1 & 1,0 & 0 & 0 & 1,0 & 0 & 0 & 1,0 & 1,46 & $\overline{1,46}$ \\
\hline 2 & 1,0 & 0 & 0 & 1,0 & 1,90 & 1,90 & 1,0 & 5,5 & 5,5 \\
\hline 3 & 1,0 & 1,23 & 1,23 & 1,0 & 2,40 & 2,40 & 1,0 & 4,64 & 4,64 \\
\hline 4 & 1,0 & 1,70 & 1,70 & 1,0 & 2,60 & 2,60 & 0,61 & 3,65 & 2,22 \\
\hline 5 & 1,0 & 1,80 & 1,80 & 1,0 & 2,80 & 2,80 & 0,32 & 3,78 & 1,21 \\
\hline 6 & 0,97 & 1,93 & 1,87 & 1,0 & 2,40 & 2,40 & 0,14 & 2,75 & 0,39 \\
\hline 7 & 0,97 & 1,86 & 1,81 & 1,0 & 2,50 & 2,50 & & & \\
\hline 8 & 0,87 & 1,58 & 1,37 & 0,90 & 2,22 & 2,0 & & & \\
\hline 9 & 0,70 & 1,33 & 0,93 & 0,80 & 0,875 & 0,70 & & & \\
\hline 10 & 0,43 & 0,85 & 0,36 & 0,40 & 0.75 & 0,30 & & & \\
\hline 11 & 0,23 & 0,86 & 0,20 & 0,20 & 1 & 0,20 & & & \\
\hline 12 & 0,10 & 2 & 0,20 & 0,10 & 0 & 0 & & & \\
\hline 13 & 0,10 & 1,70 & 0,17 & & & & & & \\
\hline 14 & 0,10 & 1 & 0,10 & & & & & & \\
\hline 15 & 0,03 & 0 & 0 & & & & & & \\
\hline $\mathrm{R}_{\mathrm{o}}$ & & & $\begin{array}{l}11,7 \\
0,9(31\end{array}$ & & & $\begin{array}{l}17,8 \\
-1,5(10)\end{array}$ & & & $\begin{array}{l}5,4 \\
3(28)\end{array}$ \\
\hline
\end{tabular}

Tableau VI a. - Taux de survie $\left(l_{x}\right)$, de fécondité $\left(m_{v}\right)$ avec l'âge ( $x$ ) et taux net de reproduction $\mathrm{R}_{n} \pm \mathrm{S}_{\mathrm{m}}$ ( $\mathrm{n}$ ) chez $B$. dimidiatus nourri de Synechoccus cedrorum, à 3 températures.

Survival rate $\left(I_{v}\right)$, age-specific fecundity-rates $\left(m_{v}\right)$ and net reproduction rates $\left(\mathbf{R}_{6}\right)$ in $B$. dimidiatus when reared at 3 temperatures with $S$. cedrorum.

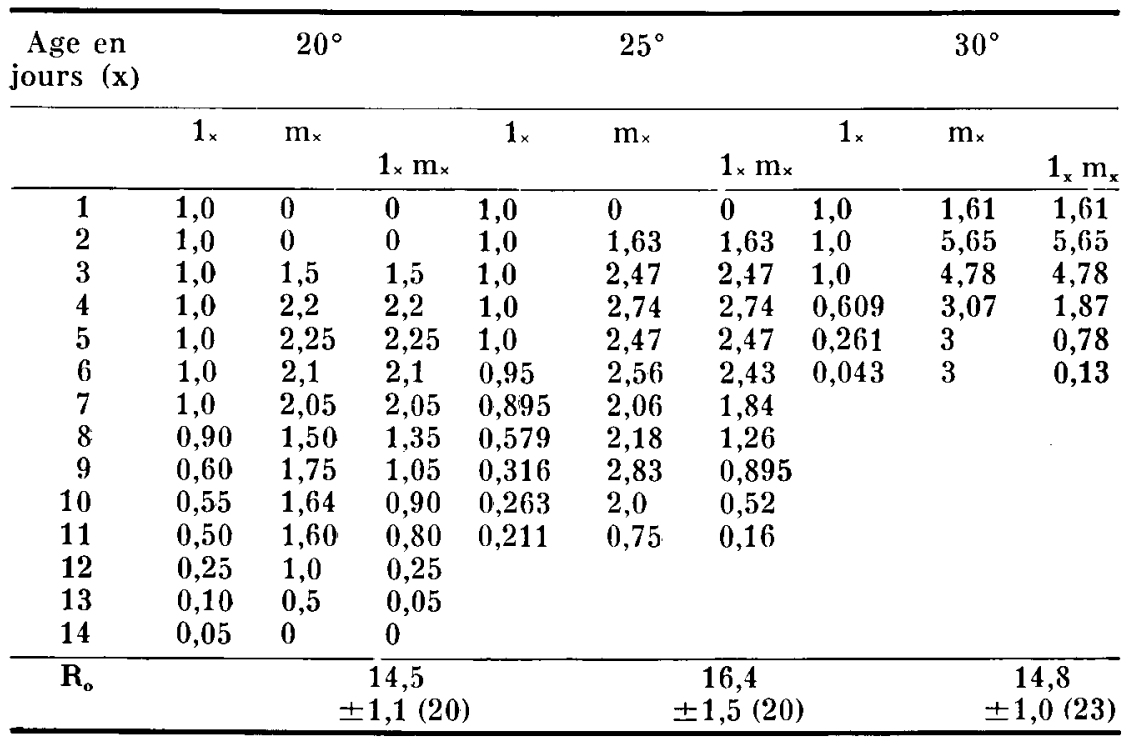

Tablead VI b. - Valeurs de $l_{x}, m_{r}$ et $\mathbf{R}_{t,}$ chez $B$. dimidiatus nourri de Dunaliella salina, à 3 températures.

Values of $l_{v}, m_{x}$ et $R_{n}$ in $B$. dimidiatus when reared at 3 temperatures with $D$. salina. 

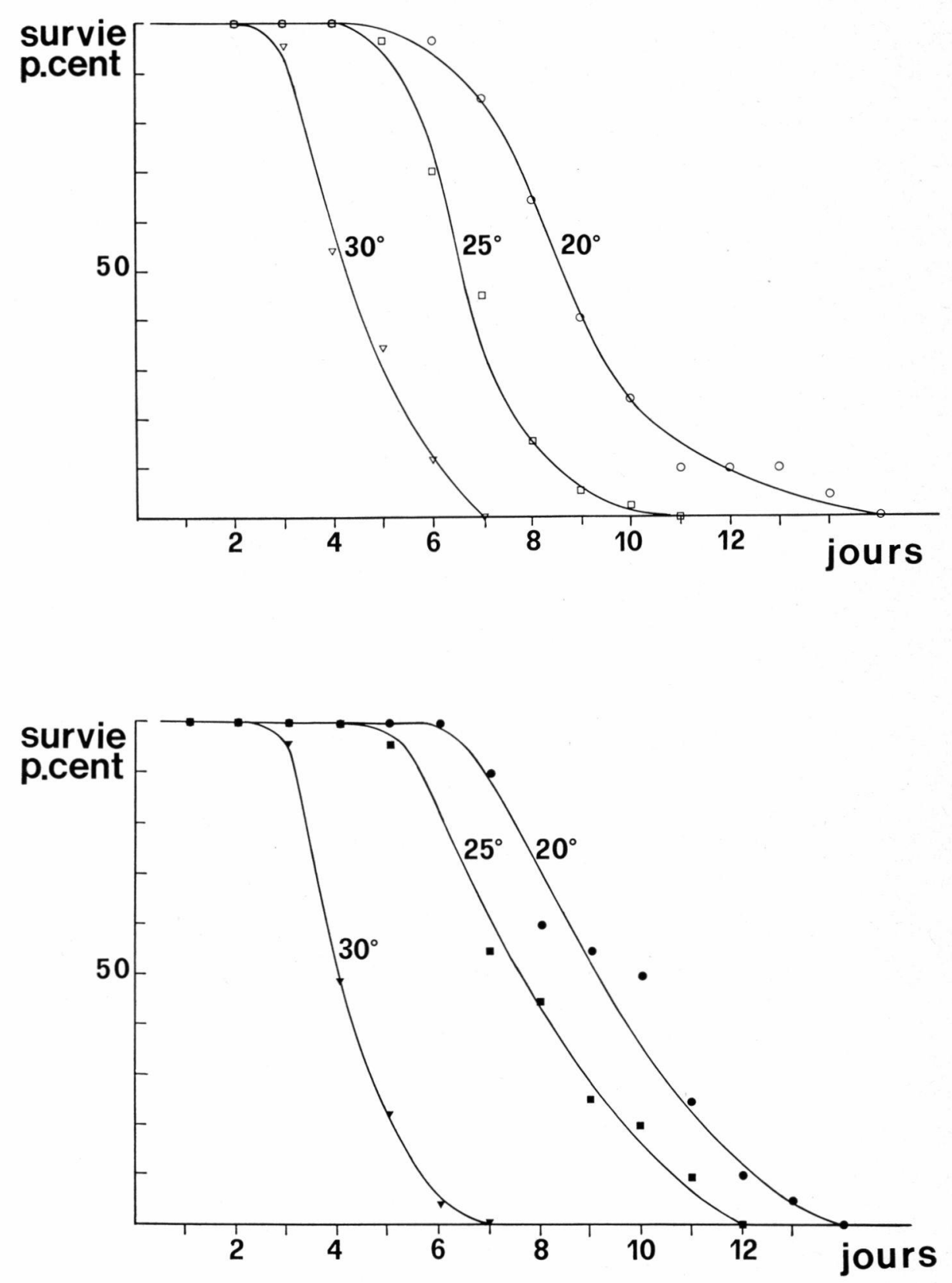

Fig. 5. - Taux de survie avec l'âge chez $B$. dimidiatus élevé dans des conditions variées de température et de nourriture avec $S$. cedrorum (5 a) ou D. salina (5 b).

Survival rates with age in $B$. dimidiatus reared in various conditions of temperature and food ( $S$. cedrorum, fig. 5 a $D$. salina, fig. 5 b). 

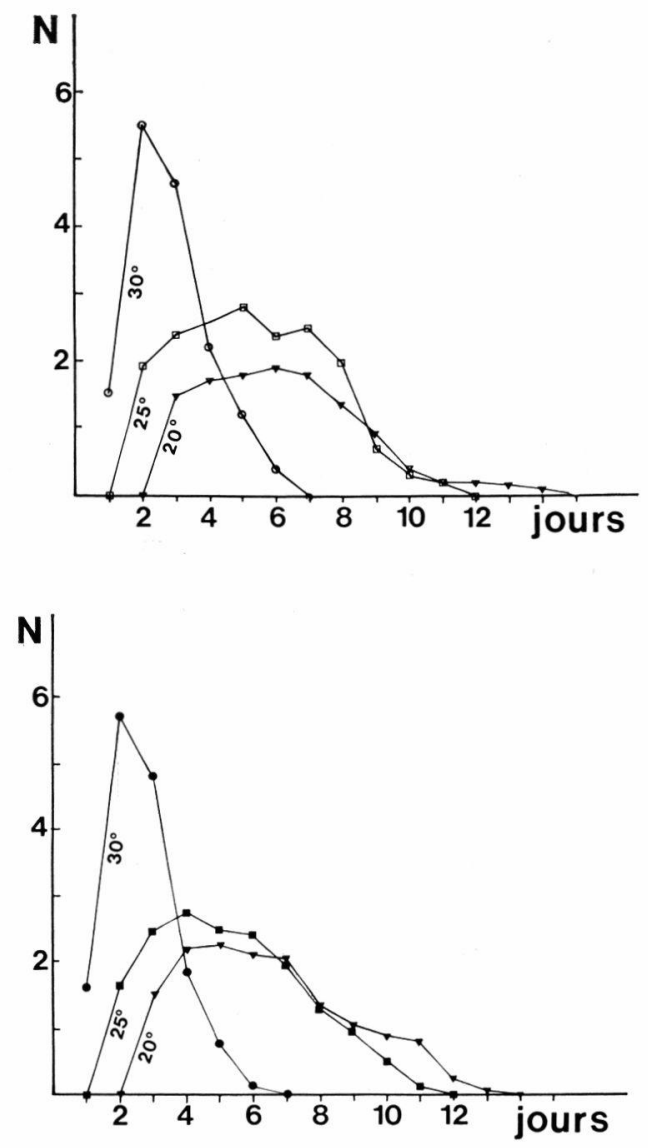

Fig. 6 - Courbes de fécondité en fonction de l'âge de la mère chez $B$. dimidiatus à 3 températures et avec 2 algues nutritives : Synechococcus cedrorum (a) et Dunaliella salina (b).

Age specific fecundity curves for $B$. dimidiatus at 3 temperatures and with 2 food species $S$. cedrorum (a) and D. salina (b).

de jours; à $25^{\circ} \mathrm{C}, 6$ jours et à $30^{\circ} \mathrm{C}, 3$ à 4 jours, c'est-à-dire deux fois moins longtemps qu'à $20^{\circ} \mathrm{C}$ (fig. 5 a et b). Ceci explique que, bien que le nombre moyen de jeunes femelles produites chaque jour soit beaucoup plus élevé à $30^{\circ} \mathrm{C}(4$ jours $)$ qu'à $20^{\circ} \mathrm{C}$ (presque 2 jeunes), Ro ait sensiblement la même valeur (tableau VII).

Une analyse de variance à deux facteurs montre que la nourriture n'a pas d'influence significative $\left(\mathrm{F}<0,1\right.$ pour $\mathrm{F}^{1}{ }_{114}=$ $3,9)$ sur Ro alors que l'influence de la température est significative $\left(\mathrm{F}=4,8\right.$ pour $\left.\mathrm{F}^{2}{ }_{114}=3,07\right)$. Il n'y a pas d'interaction entre ces deux facteurs $\left(\mathrm{F}=1,45\right.$ pour $\left.\mathrm{F}^{2}{ }_{114}=3,07\right)$. 
Considérée seule, l'action de la température ne se manifeste qu'à $20^{\circ} \mathrm{C}$ avec Synechococcus cedrorum, où la valeur de Ro est significativement plus basse que les valeurs obtenues à 25 et $30^{\circ} \mathrm{C}$. Aucune différence significative n'apparaît avec Dunaliella salina $\left(\mathrm{F}=0,7\right.$ pour $\left.\mathrm{F}^{3}{ }_{59}=2,76\right)$.

Il semble, de plus, que le nombre maximum d'œufs pondus par une femelle durant sa vie soit inféricure à $20^{\circ}$ où il n'est que de 24 à 325 alors qu'il atteint 30 à 31 oufs $/ q$ à $25^{\circ}$ et $30^{\circ}$, quelle que soit la nourriture fournie aux $B$. dimidiatus.

\subsection{Taux intrinsèque d'accroissement.}

Cet indice résume le potentiel biotique d'une population et exprime sa capacité de croissance dans un environnement illimité (croissante expotentielle), Il est défini par la constante $\mathbf{r}_{m}$ dans l'équation $N_{t}=$ No $e^{r m t}$ (Andrewartha et Birch 1954).

Le taux intrinsèque d'accroissement peut être observé sur une population en croissance exponentielle par comptages successifs de celle-ci $N_{1}, N_{2}, N_{3}$, à des intervalles de temps $t_{1}, t_{2}, t_{3} \ldots$ Ce taux peut être obtenu directement par calcul à partir de ces données :

$$
r^{\prime}=\frac{\ln N_{2}-\ln N_{1}}{t_{2}-t_{1}}
$$

Différentes formules ont été proposées pour calculer le taux intrinsèque d'accroissement à partir de tables de vie (tableau VI a et b). L'équation $\Sigma \mathrm{e}^{-\mathrm{rx}} \mathrm{l}_{\mathrm{x}} \mathrm{m}_{\mathrm{x}}=1$ (Birch 1948) permet de calculer $r_{m}$ par approximations successives en choisissant la valeur pour laquelle la somme trouvée est la plus proche de 1 (David et Fouillet 1971).

Deux valeurs approchées peuvent être obtenues, l'une proposée par Laughlin (1965) considérant $r_{c}=\frac{\ln \text { Ro }}{T}$ où $T$ représente l'intervalle moyen de génération, l'autre proposée par Parise (1966) en appliquant la formule :

$$
r_{2}=\frac{\left.R_{0}\left(\Sigma x u_{x}-\sqrt{\Sigma^{2} x} \overline{u_{x}-2\left(\Sigma x^{2} u_{x} R_{u}-\Sigma^{2} x\right.} \overline{u_{x}}\right) \text { In } R_{0}\right)}{\Sigma x^{2} u_{x} R_{0}-\Sigma^{2} x u_{x}}
$$

où $\mathrm{u}_{\mathrm{x}}=\mathrm{l}_{\mathrm{x}} \mathrm{m}_{\mathrm{x}}$.

La comparaison de ces différentes évaluations de $r$ aux trois températures expérimentées (tableau VII et fig. 7) montre une bonne concordance entre les valeurs de $r_{m}$ et les valeurs observées $\mathrm{r}^{\prime}$ particulièrement avec Synechococcus cedrorum. Les valeurs fournies par $r_{2}$ sont surestimées et celles fournies par $r_{c}$ sous- 
estimées ainsi que l'ont précédemment noté Parise (1966) et Halbach (1970). Aux températures élevées $\left(25,30^{\circ}\right)$, aucune de ces deux estimations n'est satisfaisante.

Le taux intrinsèque d'accroissement naturel s'élève avec la température. Cependant, ainsi que l'indique la figure 7, il n'apparaît pas, dans l'intervalle de température considérée $\left(20-30^{\circ}\right)$, de relation linéaire entre le taux intrinsèque d'accroissement et la température. Il en est de même pour les valeurs de $r$ obtenues à 15,20 et $25^{\circ}$ pour une population expérimentale de Brachionus calyciflorus étudiée par Halbach (1970), alors que les valeurs obtenues pour des populations naturelles montrent une telle relation (fig. 9 de Halbach 1970). La comparaison des valeurs

\begin{tabular}{lcccc}
\hline \multirow{2}{*}{ Nourriture } & $\begin{array}{c}\text { Taux intrinsèque } \\
\text { d'accroissement }\end{array}$ & $20^{\circ}$ & $25^{\circ}$ & $30^{\circ}$ \\
\hline \multirow{3}{*}{$\begin{array}{c}\text { Synechococcus } \\
\text { cedrorum }\end{array}$} & $\mathrm{r}_{\mathrm{c}}$ & 0,39 & 0,53 & 0,97 \\
& $\mathrm{r}_{2}$ & 0,50 & 0,89 & 1,59 \\
& $\mathrm{r}_{\mathrm{m}}$ & 0,45 & 0,70 & 1,25 \\
Dunaliella & $\mathrm{r}^{\prime}$ & 0,46 & 0,74 & 1,22 \\
salina & $\mathrm{r}_{\mathrm{c}}$ & 0,41 & 0,53 & 1,01 \\
& $\mathrm{r}_{2}$ & 0,56 & 0,95 & 1,46 \\
& $\mathrm{r}_{\mathrm{m}}$ & 0,50 & 0,70 & 1,28 \\
& $\mathrm{r}^{\prime}$ & 0,52 & 0,70 & 1,04 \\
\hline
\end{tabular}

Tableau VII. - Evaluation du taux intrinsèque d'accroissement naturel selon différentes méthodes, à 3 températures et avec 2 nourritures différentes. Calculation of the intrinsic rate of increase by different methods for $B$. dimidiatus reared in various conditions (3 temperatures and 2 food species).

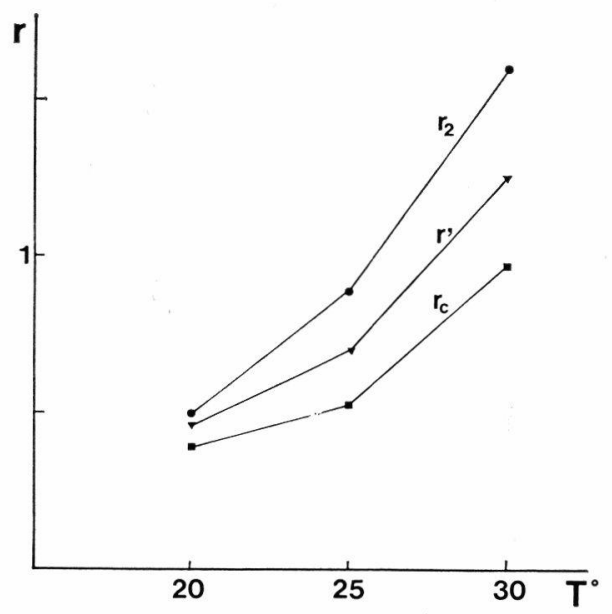

Fig. 7. - Variation des taux d'accroissement calculés de B. dimidiatus avec la température.

Variation of the calculated rate of increase of $B$. dimidiatus with the temperature. 
expérimentales et des valeurs «naturelles» de $\mathbf{r}$ permet d'expliquer cette différence. (Lorsque les valeurs expérimentales concordent bien avec les valeurs observées sur le terrain, la température peut être considérée comme le facteur limitant $\left(15,20^{\circ}\right)$. Ce ne devrait plus être le cas à $25^{\circ}$ où les taux de croissance des populations naturelles sont tous inférieurs au taux intrinsèque d'accroissement observé en laboratoire.

La relation entre taux d'accroissement (ou temps de doublement de la population) et température est linéaire chez $\mathrm{B}$. plicatilis où la valeur de $\mathrm{r}$ atteint un maximum à $27^{\circ} \mathrm{C}$ (RuttnerKolisko 1972; Hirayama et Kusano 1972).

Un point intéressant est la relation existant entre les différentes valeurs de $\mathrm{r}^{\prime}$ (taux intrinsèque d'accroissement mesuré sur une population en croissance exponentielle) et le temps minimum de génération ou plutôt son inverse puisqu'ils varient en fonction inverse l'un de l'autre. Chez $B$. dimidiatus, la relation apparaît linéaire et les équations des droites diffèrent avec la nourriture utilisée (fig. 8) :

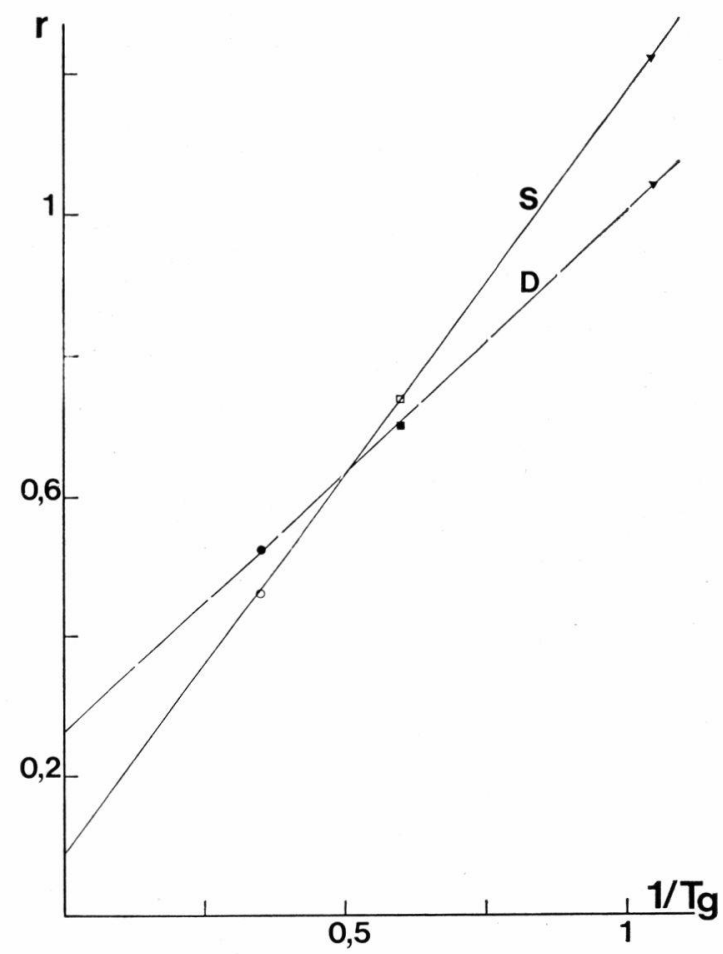

FIG. 8. - Variation des taux intrinsèques de croissance mesurés chez $B$. dimidiatus en fonction de $1 / \mathrm{Tg}$, avec 2 nourritures différentes : $S$. cedrorum (S) et D. salina (D).

Variation of the intrinsic rate of natural increase measured in $B$. dimidiatus related to $\mathrm{l} / \mathrm{Tg}$, with 2 food species : $S$. cedrorum (S), D. salina (D). 
— avec Synechococcus cedrorum, $\mathrm{r}^{\prime}=\frac{1,08}{\mathrm{Tg}}+0,08$

__ avec Dunaliella salina, $\mathbf{r}^{\prime}=\frac{0,74}{\mathrm{Tg}}+0,26$

Les temps de génération obtenus avec chaque algue nutritive étant voisins pour une température donnée (tableau IV), cette différence est vraisemblablement due aux écarts observés entre les taux nets de reproduction (Ro) dont $r^{\prime}$ dépend également : à $20{ }^{\circ} \mathrm{C}$ le taux net de reproduction est significativement inférieur avec $S$. cedrorum; à $30^{\circ}$, bien qu'il soit légèrement supérieur avec $S$. cedrorum, la différence n'est cependant pas significative.

Il semble donc que la qualité de la nourriture puisse influer sur le taux de croissance en agissant sur le seul taux net de reproduction mais non sur le temps de génération.

\section{CONCLUSION}

La comparaison des durées de développement (De, Dp el Tg) de $B$. dimidiatus avec celles d'espèces proches mais de régions tempérées ( $B$. calyciflorus, Epiphanes brachionus, Pourriot et Deluzarches 1971) fait apparaître qu'à température identique, les valeurs obtenues sont plus longues chez la première. Les durées de développement de $B$. dimidiatus à $25^{\circ} \mathrm{C}$ sont très voisines de celles de $B$. calyciflorus et de $E$. brachionus à $20^{\circ} \mathrm{C}$. Cette translation traduit l'adaptation de $B$. dimidiatus aux températures assez élevées des eaux tropicales de la zone soudanienne. De même le taux de croissance à $20^{\circ}$, relativement faible, de $B$. dimidiatus est en rapport avec sa capacité de reproduction à $30^{\circ}$, que n’ont pas les espèces des régions tempérées.

De plus, quelle que soit la température maximum tolérée par l'espèce, il apparaît un seuil inférieur pour le temps minimum de génération (environ $23 \mathrm{~h}$ ) au-dessous duquel le métabolisme est perturbé.

La variation du taux de croissance avec la température est bien plus due à l'influence de celle-ci sur les durées de développement embryonnaire et post-embryonnaire qu'à son action sur le taux net de reproduction (les valeurs de $R_{0}$ obtenues aux trois températures avec $D$. salina ne sont pas significativement différentes). Le taux net de reproduction dépend plus de la qualité (et de la quantité) de la nourriture que de la température.

A $30^{\circ}$, l'influence de la température est plus forte sur le temps de développement post-embryonnaire que sur le temps de déve- 
loppement embryonnaire. A l'inverse de ce qui se produit aux températures moins élevées, l'accélération de $D p$ contribue plus au raccourcissement du temps minimum de génération que celle de De. Ceci se traduit par un $Q_{10}$ identique, voire même supérieur (dans le cas de l'alimentation avec Synechococcus) entre 25-30 qu'entre $20-25^{\circ}$ pour $\mathrm{Dp}$ et un rapport $\mathrm{Dp} / \mathrm{De}$ à $30^{\circ}$ nettement inférieur.

L'influence du temps de développement embryonnaire (De) sur lo taux de croissance ( $r$ ) a été montré par Lewontin (cité par Birky et Gilbert 1971); celle du temps de développement postembryonnaire $(\mathrm{Dp})$, qui détermine le début de la période reproductrice semble d'égale importance et il apparaît nécessaire de lier, à l'instar de Parise (1966) le taux de croissance au temps minimum de génération qui intègre les deux paramètres précédents.

\section{TRAVAUX CITES}

Amrex (H.). 1964 (a). - Ecological studies of zooplancton in some ponds on Spitsbergen. Zool. Bid. Uppsala, 36 : 161-191.

Andrewartha (H. G.) et Birch (L. C.). 1954. - The distribution and abundance of animals. Chicago Univ. Press, 782 p.

Birch (L. C.). 1948. - The intrinsic rate of natural increase of an insect population. J. anim. Ecol., $17: 15-26$.

Birky (C. W.), Gilbert (J. J.). 1971. - Parthenogenesis in Rotifers : the control of asexual and sexual reproduction. Amer. Zool., 11 : 245-266.

D.dvid (J.), Fouillet (P.). 1971. - Le taux intrinsèque d'accroissement naturel chez Drosophila melanogaster : intérêt de ce paramètre pour les études de dynamique des populations. Terre et Vie, 3 : 378-394.

Edmonson (W. T.). 1960. - Reproductive rates of Rotifers in natural populations. Mem. Ist. Ital. Idrobiol., 12 : 21-77.

Edmonson (W. T.). 1965. - Reproductive rate of planktonic Rotifers as related to food and temperature in nature. Ecol. Monogr., $35: 61$ 111.

Gras (R.) et Saint-Jean (L.). 1969. - Biologie des Crustacés du lac Tchad. I. Durées de développement embryonnaire et post-embryonnaire: premiers résultats. Cah. O.R.S.T.O.M., sér. Hydrobiol., III (3-4) : 43-60.

Halbach (U.). 1970 a, - Einfluss der temperature auf die Populationsdynamik des planktischen Râdertiere Brachionus calyciflorus Pallas. Oecologia, $4:$ 176-207.

Hirayama (K.), Kusano (I.). - - Fundamental studies on physiology of Rotifer for its mass culture. II. Influence of water temperature on population growth of Rotifer. Bull. jap. Soc. Sci. Fish., 38 (12) : 1357-1363.

ILtis (A.). 1969. - Phytoplancton des eaux natronées du Kanem (Tchad). 1. Les lacs permanents à spirulines. 2. Les mares temporaires. Cah. O.R.S.T.O.M., sér. Hydrobiol., III (2) : 29-44 et III $(3-4): 3-20$. 
Iltis (A.), Rrou-Duwat (S.). 1971. - Variations saisonnières du peuplement en Rotifères des eaux natronées du Kanem (Tcharl). Cah. O.R.S.T.O.M., sér. Hydrobiol., 5 (2) : 101-112.

KING (Ch. E.). 1972. - Adaptation of Rotifers to seasonal variation. Ecology, 53 (3) : 408-418.

Laughis (R.). 1965. - Capacity for increase: a useful population statistic. J. anim. Ecol., 34 : 77-91.

Parise (A.). 1966. - Ciclo sessuale e dinamics di popolazioni di Euchlanis (Rotatoria) in condizioni sperimentali. Arch. Oceanogr. Limnol., 14 (3) : 387-411.

Pourriot (R.). 1965. - Recherches sur l'écologie des Rotifères. Vie Milieu, suppl. $21: 224$ p.

Pourriot (R.). 1966. - Métabolites externes et interactions biochimiques chez les organismes aquatiques. Année biol., 5 (7-8) : 337-374.

Pourriot (R.) et Deluzarches (M.). 1971. - Recherches sur la biologie des Rotifères. II. Influence de la température sur la durée du développement empbryonnaire et post-embryonnaire. Annls Limnol., 7 (1) : 25-52.

Pourriot (R.) et Hillbricht-Iljowska (A.). 1969. - Recherches sur la biologie de quelques Rotifères planctoniques. I. Résultats préliminaires. Bull. Soc. Zool. Fr., 94 (1) : 111-118.

Pourriot (R.), IltTis (A.) et LÉvếque-Duwat (S.). 1967. - Le plancton des mares natronées du Tchad. Int. Revue ges. Hydrobiol., 52 (4) : 535-543.

Rao (K. P.) et Bullock (T. H.). 1954. - Q10 as a function of size and habitat temperature in poïkilotherms. Amer. Natur., 88 (838) : $33-44$.

Rutrner-Kolisko (A.). 1972. - Der Einfluss von Temperature und Salzgehalt des Mediums auf Stoff wechsel und Vermehrungsintensität von Brachionus plicatilis (Rotatoria). Verhandl. deusch. Zool. Gesells., 65 : 89-95. 